habe, nämlich auf den dominanten, kleinbürgerlichen, leistungsorientiert-ständisch denkenden „Neuen Managertypus“ mit einem Gesellschaftsbild von individualisierten Arbeitnehmern (zu dem er Sigmar Gabriel, Thomas Oppermann, Olaf Scholz, Frank-Walter Steinmeier und Brigitte Zypries rechnet) und auf den modernisierten gewerkschaftsnahen „Arbeitnehmertypus" mit dem skandinavischen Gesellschaftsbild eines solidarischen Arbeitnehmers (Andrea Nahles). Wo die Sympathien des Verfassers liegen, ist offenkundig: bei der linken Faktion. Allerdings bedauert er, dass die heutige SPD-Linke keine systemüberwindenden Positionen vertrete und letztlich zu einem Mitte-Links-Bündnis geworden sei. Ihr fehlten die entschiedenen Sozialisten, die Kurt Schumacher noch integriert habe. Mit besonderer Zuneigung behandelt Reinhardt sein Idol Peter von Oertzen, erweckt fast den Eindruck, als seien dessen rätesozialistische Positionen zeitweilig in einer Faktion der SPD vertreten worden.

Abschließend stellt Reinhardt fest, dass die SPD heute wirtschaftsliberal verengt sei, ihr rechter Flügel die Partei dominiere. Er fordert, dass die SPD wieder milieuübergreifende und -integrierende Volkspartei werden müsse. Diese Forderung bleibt recht voluntaristisch, struktureller Wandel wird nur unzureichend bedacht: Veränderungen zwischen sekundärem und tertiärem Sektor; Ende der Arbeiterkulturbewegung und damit des entsprechenden Milieus; Ende des Organisationsnetzwerkes, das die Partei und ihre Kultur- und Freizeitvereine einst zusammenhielt. Das Ende der Volksparteien, so, wie wir sie aus der Geschichte kennen, dürfte endgültig gekommen sein - und damit auch das der Volkspartei SPD.

Um nicht missverstanden zu werden: Die normativen Positionen und auch Zuspitzungen Reinhardts sind nicht negativ zu kritisieren, vielmehr positiv hervorzuheben. In einem Zeitalter, in dem Doktoranden sich brav an ihre akademischen „Väter" und „Mütter“ anpassen, ist eine Dissertation wie die vorliegende erfrischend, reizt zum Widerspruch oder zur vehementen Zustimmung. Dies spricht für den Autor, Max Reinhardt, und seinen Betreuer, Michael Vester. Was mehr irritiert, ist das Auseinanderfallen des Bandes in zwei Darstellungen.

Peter Lösche

\title{
CDU: Strategien der Machterhaltung und innere Spannungsfelder auf dem Weg in die Mitte
}

Walter, Franz, Christian Werwath und Oliver d'Antonio: Die CDU. Entstehung und Verfall christdemokratischer Geschlossenheit (Schriftenreihe „Die politischen Parteien der Bundesrepublik Deutschland“), Nomos Verlagsgesellschaft, Baden-Baden 2011, 261 Seiten, € 19,90.

Neumann, Arijana: Die CDU aufLandesebene. Politische Strategien im Vergleich, VS Verlag für Sozialwissenschaften, Wiesbaden 2012, 322 Seiten, $€$ 39,95.

Die Machtstrukturen der Parteien und ihre Strategien im Wettbewerb um Wählerstimmen werden am Beispiel der CDU in zwei neu erschienenen Arbeiten untersucht. Während bei Franz Walter, Christian Werwath und Oliver d'Antonio die Anpassung der CDU an veränderte gesellschaftliche Rahmenbedingungen im Zeitverlauf betrachtet wird, analysiert Arijana Neumann Einflussfaktoren einer erfolgreichen Regierungsübernahme der CDU in den Ländern während der Regierungszeit von SPD und Grünen auf Bundesebene. 
Die Untersuchung von Franz Walter, Christian Werwath und Oliver d'Antonio ist der erste Band einer von Karl-Rudolf Korte herausgegebenen Reihe: „Die politischen Parteien der Bundesrepublik Deutschland“, deren Ziel die „systematische Darstellung der Wirkungsgeschichte, der internen Entscheidungsprozesse und der strategischen Entwicklungspotenziale der CDU, der SPD, der FDP, von Bündnis 90/Die Grünen und der Partei Die Linke“ ist (S. 5). In jedem Band soll die „Frage nach der formalen und informellen Organisation der internen Entscheidungsprozesse“ und den „internen Machtzentren der Partei“ beantwortet werden (S. 6). Ein nachvollziehbares Interesse, zeigt sich doch trotz sinkender Parteibindungen und nachlassender Prägekraft sozialmoralischer Milieus ein erstaunliches Beharrungsvermögen der etablierten Parteien im deutschen Parteiensystem. Weder sich ausdünnende katholische Wählermilieus oder die abnehmende Kirchenbindung noch das Anwachsen säkularisierter Arbeitnehmerschichten und postmaterialistischer Einstellungsmuster haben bisher etwas an der relativen Vormachtstellung der Union geändert (S. 11) - ein Befund, der durchaus nicht selbstverständlich ist, wenn man bedenkt, dass viele der heute über 60 -jährigen westdeutschen Wähler mit der so genannten Studentenrevolte und damit verbundenen Diskursen zumindest mittelbar in Berührung gekommen sein dürften (S. 169).

Walter, Werwath und d'Antonio sehen die historischen Ausgangsbedingungen des Erfolges der CDU im Gründungsmythos der Partei, verstanden als die Ineinssetzung der Wohlstandsmehrung in den Anfangsjahren der Bundesrepublik mit dem politischen Agieren der CDU (S. 16). Die Partei habe es gerade unter der Führung Konrad Adenauers verstanden, das Lebensgefühl breiter gesellschaftlicher Schichten der Bonner Republik zu verkörpern, denn nach der Diktaturerfahrung hätten sich die Deutschen gesehnt nach „Ruhe, Sicherheit und einem erträglichen Auskommen, wollten politisch entpflichtet sein“ (S. 15). Dem entspräche der Unionsansatz einer bewusst weitgehend „illusionslosen, pragmatischen und flexiblen Politik“ (S. 17). Mit ihrem Selbstverständnis als überkonfessionelle Sammlungsbewegung auf christlichem Wertefundament habe die Union über „ein flexibles und überzeitlich ausgerichtetes Werte-Set“ verfügt (S. 17). Das christliche Menschenbild sei das Kernstück des „Unionsgedankens" und diene als einigende Klammer ihrer Politik (S. 65). Es ist Teil des christlichdemokratischen Selbstverständnisses, das „Zentrum des Parteiensystems“ zu repräsentieren und von dort aus als „Scharnierstelle bei der Koalitions- und Regierungsbildung “ zu fungieren (S. 19). Obgleich der christdemokratische Konservatismus weder zu „großen Visionen “ noch zu „Utopien“" neige, sei er durch die Rückbindung an die gesellschaftliche Mehrheitsmeinung "ganz und gar empirisch“ (S. 21). Schon in den Anfangsjahren habe es der Union daher nicht unbedingt geschadet, sondern eher genutzt, dass „die Milieus ihre starren Grenzen nicht mehr hatten ziehen können, dass sie offener, durchlässiger, weniger dogmatisch, integrationsbereiter waren. Denn [...] erst dadurch konnte eine Einheitspartei des katholisch-protestantischen Bürgertums in Deutschland reüssieren“ (S. 24). Mit der zunehmenden Entvölkerung der christlichen Vorhöfe der Union sei es dann zur Besinnung auf ein Modell des „von intellektuellen Zweifeln an den Grundaxiomen ihrer Politik entkleidete[n] Pragmatismus und [einer] Strategie der Pazifizierung des Souveräns" gekommen (S. 54). Wenn es um Macht und Einfluss ging, blieb die Union trotz einer sich verschlechternden Ausgangssituation stets eine „hochelastische, geschmeidig anpassungsfähige Partei“" (S. 69).

Vor dem Hintergrund dieser Analyse sind die Ausführungen zu den inneren Spannungsfeldern der CDU besonders lesenswert. Die Autoren sehen Anzeichen einer strategisch begründeten Universalisierung des Mittebegriffs und dessen Kappung von ihren rein bürgerlichen Wurzeln (S. 204). Für diese These spricht unter anderem, dass sich die CDU von der wirt- 
schaftsliberalen und freiheitlichen Akzentuierung des Leipziger Parteitags von 2003 erkennbar verabschiedet hat (S. 152). Es ist zwar durchaus fraglich, ob sie gleich die „neuliberalen Versatzstücke aus dem Parteidenken [...] verbannen und sich verstärkt [an] einer volksnäheren, kleinbürgerlichen Interessenstruktur orientieren [sollte]", wie Walter, Werwath und d'Antonio raten (S. 198). Zwar könnte dies den Christdemokraten mehr Koalitionsmöglichkeiten im Parteienspektrum eröffnen (S. 216). Fraglos aber bliebe eine solche programmatische Positionierung nicht ohne Auswirkungen auf diejenige Strömung, die sich in der Tradition der klassischen Ordnungspolitik und ihrer akademischen Vordenker sieht (S. 152). Denn ob „die Mitte“ gänzlich auf die Integration eines von der ökonomischen Theorie gedeckten Denkens und der Artikulation damit verbundener Grundprinzipien wie der Eigenverantwortung, der Zusammengehörigkeit von Risiko, Haftung und solider Haushaltsführung verzichten kann, ist durchaus fraglich. Die Vielfalt der unter ihrem Banner versammelten Meinungen ist für die CDU eben nicht nur Chance, sondern auch fortwährende Herausforderung (S. 221 f.).

Im Unterschied zur in erster Linie deskriptiven Darstellungsweise von Walter, Werwath und d'Antonio, deren Lektüre sich auch für den nicht wissenschaftlich vorgeprägten Leser anbietet, geht Arijana Neumann streng systematisch vergleichend vor (S. 18). Bezugspunkt ihrer Analyse ist die in der deutschen Parteienforschung noch immer stiefmütterlich behandelte regionale Ebene (S. 18). Mit ihrem dezentralen Ansatz fördert Neumann so insbesondere aufgrund des föderalen Charakters der Union interessante Gemeinsamkeiten und Unterschiede in den jeweiligen programmatischen Schwerpunktsetzungen der Partei zutage. Die Autorin untersucht vier Landesverbände der CDU, die in den Ländern während der Regierungszeit von SPD und Bündnis 90/Die Grünen im Deutschen Bundestag die Regierungsverantwortung übernommen haben (S. 19). Aufgrund des „most-similar-cases-designs“ findet, wie Neumann selbst bedauert, zwar kein ostdeutsches Land Berücksichtigung (S. 49). So wäre beispielsweise auch ein Blick auf Sachsen-Anhalt im Jahr 2002 durchaus interessant gewesen. Dennoch wird die Fallauswahl der Länder Hessen, Saarland, Niedersachsen und Nordrhein-Westfalen logisch begründet (S. 48 ff.).

Grundlegend für die Arbeit ist die Annahme, dass eine notwendige (wenngleich nicht hinreichende) Bedingung einer erfolgreichen Regierungsübernahme der Oppositionspartei die Existenz eines verschiedene Schnittstellen übergreifenden "strategischen Zentrums" ist (S. 39 f.). Hinzutreten sollten sogenannte „critical junctures“ im Zuge der jeweiligen Landtagswahlen. Gemeint sind damit nicht alltägliche Begleitumstände aus Sicht der Opposition, die sich nicht unmittelbar aus dem parlamentarischen Zusammenspiel der Kräfte ergeben haben müssen, wohl aber die Mobilisierungsfähigkeit der Opposition entscheidend erhöhen. Hierzu zählen von der Opposition nur mittelbar zu beeinflussende Faktoren wie die Zufriedenheit mit der aktuellen Bundesregierung und der Landesregierung ebenso wie unmittelbar zu beeinflussende Faktoren wie die Erarbeitung eines besonderen inhaltlichen Kompetenzvorsprungs und erfolgversprechender Persönlichkeitswerte beim Spitzenpersonal (S. 43).

Die inhaltliche Bandbreite in der CDU wird durch die regionale Perspektive in unterschiedlichen Akzentsetzungen bei der Ausrichtung des Wahlkampfs, aber auch nach der Regierungsübernahme deutlich. So habe beispielsweise der traditionell eher konservativen Hessen-CDU mit der von der rot-grünen Bundesregierung diskutierten „doppelten Staatsbürgerschaft" ein Thema zur Verfügung gestanden, mit dem das eigene Lager mobilisiert werden konnte (S. 75). Einmal in Regierungsverantwortung, profilierte sich die hessische CDU dann jedoch eher als wirtschaftsliberale, kompetitiv-föderalistische Alternative zur Bun- 
desregierung (S. 105). Auch die saarländische CDU habe ihre Wahlaussichten mithilfe einer Unterschriftenkampagne zur Rentenpolitik der rot-grünen Bundesregierung zu verbessern versucht (S. 120). In der Regierung sei es ein Ziel gewesen, sich durch sozialpolitische Absetzbewegungen von den Beschlüssen des Bundes weiter zu profilieren (S. 134). In Niedersachsen kam der CDU im Vorfeld des Wahlerfolges im Jahr 2003 der Weggang des sozialdemokratischen Spitzenpersonals nach 1998 entgegen. Die Persönlichkeitswerte des Herausforderers Christian Wulff waren im Vergleich zum sozialdemokratischen Ministerpräsidenten überdurchschnittlich gut (S. 163). In der Regierungsarbeit versuchte die CDU einen Ausgleich zwischen den ländlich-konservativen und großstädtisch-liberalen Kräften innerhalb der Partei (S. 165) herzustellen. Die traditionell sozial orientierte nordrhein-westfälische CDU habe bei der Landtagswahl 2005 vom Höhepunkt der Unzufriedenheit über die Arbeitsmarktreformen der Bundesregierung profitiert (S. 207). Den am Wahlabend verkündeten Anspruch auf die Arbeiterführerschaft konnte man aufgrund eingeschränkter Gestaltungsmöglichkeiten in der Arbeitsmarktpolitik nicht in das Regierungshandeln übertragen (S. 280).

Der Wert der Untersuchung von Arijana Neumann liegt zweifellos in der analytischen Reduktion eines sehr umfangreichen und komplexen Gegenstands mithilfe eines mehrgliedrigen regionalen Vergleichs. In dem lobenswerten Bemühen, sowohl die oppositionellen Strategien der Regierungsübernahme als auch der Machterhaltung der einstigen Oppositionspartei in vier verschiedenen Landesverbänden zu analysieren, beschreibt die Arbeit zahlreiche interessante Einzelaspekte. Leider gehen die damit verbundene Informationsfülle und zwangsläufig recht schematische Argumentation auch zulasten der analytischen Stringenz. Selbst mit einer vergleichenden Untersuchung der Regierungsübernahme in zwei Ländern hätte Neumann noch ausreichenden Forscherehrgeiz bewiesen. Das Interesse an den Strategien der Machterhaltung nach der Übernahme der Regierungsverantwortung ist zwar mit Blick auf die zentrale Bedeutung des „strategischen Kraftzentrums“ für die vorangegangene Regierungsübernahme verständlich (S. 264). Allerdings wirkt der Untersuchungsgegenstand auch dadurch etwas zu breit abgesteckt. Trotz dieser Einwände öffnet die regionale Perspektive, gerade unter den Bedingungen eines immer volatileren Wahlverhaltens, den analytischen Blick weiter für die unmittelbaren Einflüsse und das strategische Verhalten im Parteienwettbewerb.

Sebastian Rehse

\section{CSU: detaillierte Analysen, lückenhafte Themenpalette}

Hopp, Gerhard, Martin Sebaldt und Benjamin Zeitler (Hrsg.): Die CSU. Strukturwandel, Modernisierung und Herausforderungen einer Volkspartei, VS Verlag für Sozialwissenschaften, Wiesbaden 2010, 590 Seiten, € 39,95.

Die Christlich Soziale Union (CSU) befinde sich an einer „Wegscheide“, so der Journalist Georg Paul Hefty. Kann sie ihre über Jahrzehnte gefestigte Position im deutschen Parteiensystem als bayerische Regionalpartei mit bundespolitischer Gestaltungsmacht verteidigen oder büßt sie diese ein? Auch die Christsozialen verlieren im Zuge langfristiger gesellschaftlicher Entwicklungen (unter anderem Säkularisierung, demographischer Wandel, Tertiarisierung, Urbanisierung, Bildungsexpansion) Mitglieder und Wähler. Für die Analyse des 